\title{
Oral Candidiasis among Cancer Patients Attending a Tertiary Care Hospital in Chennai, South India: An Evaluation of Clinicomycological Association and Antifungal Susceptibility Pattern
}

\author{
Abirami Lakshmy Jayachandran, ${ }^{1}$ Radhika Katragadda, ${ }^{2}$ Ravinder Thyagarajan, ${ }^{2}$ \\ Leela Vajravelu, ${ }^{2}$ Suganthi Manikesi, ${ }^{2}$ Shanmugam Kaliappan, ${ }^{3}$ and Balaji Jayachandran ${ }^{4}$ \\ ${ }^{1}$ Department of Microbiology, Karpaga Vinayaga Institute of Medical Sciences and Research Center, \\ Madurantakam Taluk, Kanchipuram, Tamil Nadu 603308, India \\ ${ }^{2}$ Department of Microbiology, Government Kilpauk Medical College Hospital, Chennai, Tamil Nadu 600010, India \\ ${ }^{3}$ Department of Microbiology, King Institute of Preventive Medicine, Guindy, Chennai, Tamil Nadu 600032, India \\ ${ }^{4}$ Department of Prosthodontics, Indira Gandhi Institute of Dental Sciences, Puducherry 607402, India \\ Correspondence should be addressed to Abirami Lakshmy Jayachandran; alfeb85@gmail.com
}

Received 8 February 2016; Revised 11 May 2016; Accepted 22 May 2016

Academic Editor: Christine A. Hughes

Copyright ( 2016 Abirami Lakshmy Jayachandran et al. This is an open access article distributed under the Creative Commons Attribution License, which permits unrestricted use, distribution, and reproduction in any medium, provided the original work is properly cited.

\begin{abstract}
Oropharyngeal candidiasis is one of the common manifestations seen in cancer patients on cytotoxic therapy and invasion into deeper tissues can occur if not treated promptly. Emergence of antifungal drug resistance is of serious concern owing to the associated morbidity and mortality. The present study aims at evaluation of clinicomycological association and antifungal drug susceptibility among the 180 recruited patients with cancer on chemotherapy and/or radiotherapy with signs or symptoms suggestive of oral candidiasis. Speciation and antifungal susceptibility was done by Microbroth dilution method for fluconazole, Itraconazole, and Amphotericin B as per standard microbiological techniques. Chi-square test was used for statistical analysis ( $p<0.05$ was considered statistically significant). Candida albicans was the predominant species isolated (94) (58\%) followed by Candida tropicalis (34) (20.9\%). Fluconazole and Itraconazole showed an overall resistance rate of $14 \%$ and $14.8 \%$, respectively. All the isolates were susceptible to Amphotericin B. There was a significant association between the presence of dry mouth and isolation of Candida $(p<0.001)$. Such clinicomicrobiological associations can help in associating certain symptoms with the isolation of Candida. Species level identification with in vitro antifungal susceptibility pattern is essential to choose the appropriate drug and to predict the outcome of therapy.
\end{abstract}

\section{Introduction}

Oropharyngeal candidiasis is a common fungal infection in immunocompromised individuals. Conditions like malignancies, chemotherapy, and radiotherapy compromise the cell mediated immunity predisposing the person to fungal infections [1]. Candida species are normally present as commensals in the oral cavity and their transition to become an opportunistic infective agent may be associated with certain virulence determinants [2]. Incidence of oral candidiasis has been reported to be ranging from 7 to $52 \%$ among cancer patients (head and neck malignancy, hematopoietic malignancy, and solid tumors) on chemotherapy and or radiotherapy [1].

A higher incidence of oral colonisation with non-Candida albicans has been reported in patients with advanced stage of cancer [3]. Although Candida albicans and non-Candida albicans are closely related, they differ in the antifungal susceptibility patterns. The colonised Candida can invade the underlying mucosa and enter the blood stream leading 
onto disseminated disease with considerable morbidity and mortality if not treated promptly. Fluconazole is one of the first line drugs used for the treatment of oral candidiasis in cancer patients $[4,5]$. Amphotericin B is usually used for invasive Candida infections. Newer drugs like echinocandins are reserved for therapy of refractory candidiasis $[4,5]$. It is of vital importance that cancer patients should be evaluated clinically and microbiologically for the presence of Candida in the oral cavity. The present study aims at speciation of the Candida isolated from oral cavity of patients with malignancy, to study the antifungal susceptibility pattern of the isolates and to evaluate the association between clinical and mycological findings.

\section{Materials and Methods}

This observational study was conducted in the Department of Microbiology, Government Kilpauk Medical College, Chennai, India, during the period of one year (Jan 2013 to Jan 2014). The study was approved by the institutional ethical committee. Cancer patients on chemotherapy and/or radiotherapy attending either outpatient or inpatient oncology clinic with signs and symptoms suggestive of oral candidiasis like presence of white plaque, erythematous lesion, ulcerative lesion, dryness of mouth, pain, altered taste sensation, and halitosis were included in the study. Unwillingness to participate and patients on antifungal therapy for past two weeks were excluded from the study. The study was explained and informed consent was obtained from the patients. The demographic data, present and past clinical history (type of cancer and treatment details), and complaints like presence of white patch in the oral cavity, pain, and erythematous lesion were documented in a pro forma for each patient. The data from the pro forma were analysed and tabulated and the association between the clinical information and the mycological findings was assessed and evaluated.

Two sterile swabs were used to collect sample from the oral cavity by swabbing over the lesions. The lesions (white patch, erythematous, and ulcerative lesion) were present over the buccal mucosa, tongue, and gingival regions and over the palatal regions in some cases. One swab was used for direct gram staining to look for the presence of gram positive yeast cell and pseudohyphae. The other swab was used for inoculating the specimen into Sabouraud dextrose agar and incubated at $24^{\circ} \mathrm{C}$ for 48 hours. The growth of creamy white colonies was subjected to gram staining for presence of gram positive budding yeast cells. Colonisation is defined as the presence of yeast cells in the oral cavity with/without clinical signs and symptoms. Infection or oral candidiasis is defined as the demonstration of gram positive hyphae/pseudohyphae and yeast cells microbiologically along with clinical signs and symptoms. Germ tube test was performed for all the isolates and further speciation was done by colony morphology in chrom agar (color of the colony), growth in corn meal agar (dalmau plate culture), and sugar assimilation and fermentation test as per standard microbiological techniques [6].

2.1. Antifungal Susceptibility Testing. Antifungal susceptibility testing was performed by Microbroth dilution method using RPMI (Roswell Park Memorial Institute) Medium 1640 with glutamine as per CLSI guidelines (2009) [7, 8]. Stock suspension was prepared and diluted with RPMI Medium to obtain a final inoculum size of $1 \times 10^{3}$ to $5 \times 10^{3} / \mathrm{CFU} / \mathrm{mL}$. The stock solutions were prepared by dissolving fluconazole powder in sterile distilled water. Amphotericin B and Itraconazole were dissolved in dimethyl sulfoxide. The drug $(2 \mathrm{x}$ concentration) was dispensed into the wells of the sterile disposable Microtitre plate $(100 \mu \mathrm{L}$ volume from row 1 to 10) with the highest drug concentration in row 1 and lowest concentration in row 10 . Each well was inoculated with the $100 \mu \mathrm{L}$ of $2 \mathrm{x}$ inoculum suspension. The growth control well contains sterile drug-free medium and the corresponding inoculum suspension. The drug-free medium was added to row 11 to act as a growth control. The Microtitre plates were incubated at $35^{\circ} \mathrm{C}$ for 48 hours. The plates were observed for the presence or absence of visible growth. A numerical score which ranges from 0 to 4 was given to each well $[7,8]$ :

0 : optically clear.

1: slightly hazy or approximately $25 \%$ of growth control.

2: prominent decrease in turbidity or approximately $50 \%$ of growth control.

3: slight reduction in turbidity or approximately $80 \%$ of growth control.

4: no reduction in turbidity.

End Point of MIC. End point of MIC was considered as follows:

Fluconazole and Itraconazole; score 2 or less,

Amphotericin B: score 0.

The results are interpreted as per CLSI guidelines 2009.

2.2. Statistical Analysis. The sensitivity, specificity, positive predictive value, and negative predictive value of direct gram staining versus the culture positivity and germ tube test positivity in identifying Candida albicans were calculated. The significance of association between the symptoms/signs and the isolation of Candida was analysed by chi-square test and a two-sided $p$ value less than 0.05 was considered statistically significant (Graphpad Quick Calcs software).

\section{Results}

A total of 192 cancer patients were initially recruited out of which twelve were excluded (eight patients were unwilling to participate and the rest were on antifungal therapy). Out of the 180 patients included in the study, male patients comprised $58.3 \%$ and females encompassed 41 (Table 1). The details regarding the distribution of cases by risk factors are depicted in Table 1. Patients with oral cancer comprised the major percentage of cases followed by gastrointestinal tract (GIT) malignancy (Table 1). All the cancer patients were on either chemotherapy or radiotherapy. The clinical symptoms and signs were analysed. The most commonly 
TABLE 1: Demographic data and the distribution of patients based on cancer type.

\begin{tabular}{lc}
\hline & Number \\
\hline Age & $20(11.1 \%)$ \\
$0-20$ & $44(24.4 \%)$ \\
$21-40$ & $64(35.5 \%)$ \\
$41-60$ & $52(28.8 \%)$ \\
$61-70$ & \\
\hline Sex-wise distribution & $105(58.3 \%)$ \\
Male & $75(41.6 \%)$ \\
Female & \\
\hline Distribution of the patients based on the type of & \\
cancer & $76(42.2 \%)$ \\
Carcinoma oral cavity & $26(14.4 \%)$ \\
Carcinoma stomach & $22(12.2 \%)$ \\
Carcinoma esophagus & $16(8.8 \%)$ \\
Lymphoma & $14(7.7 \%)$ \\
Carcinoma cervix & $12(6.6 \%)$ \\
Chronic myeloid leukemia & $8(4.4 \%)$ \\
Bone tumor & $4(2.2 \%)$ \\
Carcinoma bladder & $2(1.1 \%)$ \\
Carcinoma colon & \\
\hline
\end{tabular}

encountered symptom in the present study was dryness of mouth followed by pain in the oral cavity (Figure 1). The most common sign observed was presence of white plaques/patches and redness in the mucosa of oral cavity (Figure 1). The association between clinical details such as signs, symptoms, and mycological findings was evaluated and it was found that there was a significant association between dryness of mouth and isolation of Candida from the oral cavity $(p<0.001)$ (Table 2$)$.

In the present study, $90 \%$ of the cases showed direct gram staining positivity (presence of gram positive yeast cells and pseudohyphae) (Figure 2). The specimens that are culture positive were further speciated by germ tube test (for differentiating Candida albicans from non-Candida albicans), growth in chrom agar, corn meal agar, sugar assimilation, and fermentation tests.

The sensitivity, specificity, negative predictive value, positive predictive value, and diagnostic accuracy of gram staining versus culture positivity and positivity of germ tube test to identify Candida albicans are depicted in Table 3. Gram staining showed a sensitivity of $90 \%$. Germ tube test showed sensitivity and specificity of $95 \%$. Out of the 180 specimens received $152(88.3 \%)$ were culture positive for Candida. Oral Candida infection was seen highest among patients with carcinoma of oral cavity (68) (89\%) followed by carcinoma of gastrointestinal tract (34) (68\%). Candida albicans (94) (58\%) was the predominant species isolated followed by Candida tropicalis (34) (20.9\%), Candida glabrata (14) (8.6\%), Candida krusei (10) (6.17\%), Candida parapsilosis (6) (3.7\%), and Candida kefyr (4) (2.46\%). Mixed infection (isolation of two species of Candida) was seen in 10 patients (Table 4).

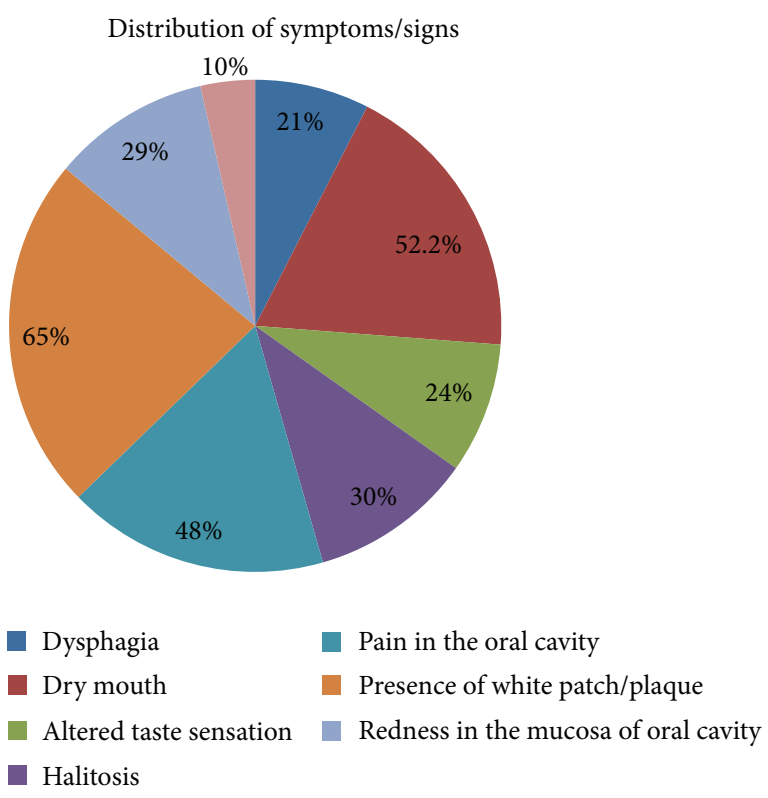

FIGURE 1: Distribution of symptoms and signs among the cancer patients.

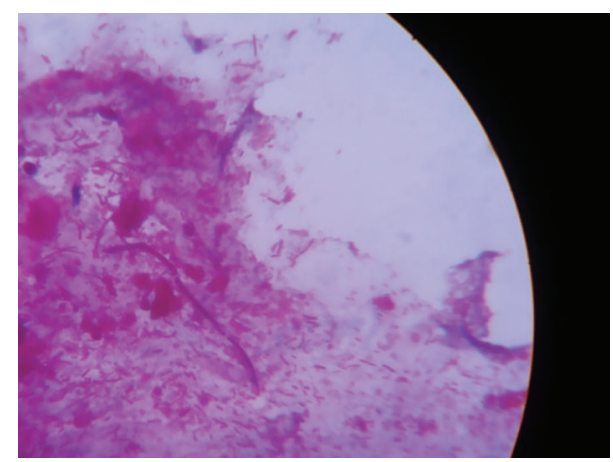

FIGURE 2: Direct gram staining showing the presence of gram positive hyphae.

Antibiotic susceptibility pattern was performed by Microbroth dilution method (Table 5). The overall resistance percentage for fluconazole and Itraconazole was $14 \%$ and $14.8 \%$, respectively. Fluconazole showed a resistance of $5.8 \%$ and $12 \%$ for Candida albicans and Candida tropicalis, respectively. Itraconazole showed a resistance of $12 \%$ for fluconazole and Itraconazole each for Candida albicans and Candida tropicalis. None of the isolates of Candida krusei was susceptible to fluconazole. Candida glabrata showed the highest resistance for fluconazole (21.5\%) and Itraconazole (14.3\%). All the isolates were susceptible to Amphotericin B. The present study has documented that non-Candida albicans showed a higher percentage of resistance compared with Candida albicans. 
TABLE 2: Mycological and clinical association between the symptoms/signs.

\begin{tabular}{|c|c|c|c|c|c|}
\hline S. number & Symptom/signs & No isolation of candida & Isolation of candida & Total & $p$ value \\
\hline \multirow{2}{*}{1} & Presence of dry mouth & 68 & 26 & 94 & \multirow{2}{*}{$<0.001$} \\
\hline & Absence of dry mouth & 84 & 2 & 86 & \\
\hline \multirow{2}{*}{2} & Presence of erythema & 41 & 11 & 52 & \multirow{2}{*}{0.187} \\
\hline & Absence of erythema & 111 & 17 & 128 & \\
\hline \multirow{2}{*}{3} & Presence of white patch & 20 & 119 & 139 & \multirow{2}{*}{0.25} \\
\hline & Absence of white patch & 7 & 44 & 51 & \\
\hline \multirow{2}{*}{4} & Presence of ulcer & 6 & 12 & 18 & \multirow{2}{*}{-} \\
\hline & Absence of ulcer & 146 & 16 & 162 & \\
\hline
\end{tabular}

TABLE 3: Sensitivity, specificity, negative predictive value, positive predictive value, and the diagnostic accuracy of (a) direct gram staining versus culture positivity and (b) germ tube test positivity in identifying Candida albicans.

\begin{tabular}{lccccc}
\hline Parameter & Sensitivity & Specificity & Negative predictive value & Positive predictive value & Diagnostic accuracy \\
\hline Direct gram staining & $90.06 \%$ & $100 \%$ & $52.63 \%$ & $100 \%$ & $91.04 \%$ \\
Germ tube test & $95.68 \%$ & $95.40 \%$ & $87.50 \%$ & $98.52 \%$ & $95.63 \%$ \\
\hline
\end{tabular}

TABLE 4: Species-wise distribution of the isolates.

\begin{tabular}{lcc}
\hline S. number & Species & Percentage \\
\hline 1 & Candida albicans & $94(58 \%)$ \\
2 & Candida tropicalis & $34(20.9 \%)$ \\
3 & Candida glabrata & $14(8.6 \%)$ \\
4 & Candida krusei & $10(6.17 \%)$ \\
5 & Candida parapsilosis & $6(3.7 \%)$ \\
6 & Candida kefyr & $4(2.46 \%)$ \\
\hline & Total & 162 \\
\hline
\end{tabular}

Mixed infection was seen in 10 patients (isolation of two Candida species).

\section{Discussion}

Oral candidiasis is a major problem in the world especially among cancer patients on cytotoxic therapy. The prevalence of oropharyngeal candidiasis was reported to be $38 \%$ by Ramirez-Amador et al. among cancer patients on radiotherapy [9]. Studies have reported the incidence of oral candidiasis to be ranging from 7 to $52 \%$ in cancer patients on chemotherapy and/or radiotherapy [1]. Patients usually progress from asymptomatic colonisation stage to infection. Conditions like malignancy, chemotherapy, and radiotherapy compromise the immunity and make the patient vulnerable to oropharyngeal candidiasis. The various other risk factors are use of antibacterials and steroids, comorbid illness like diabetes, poor oral hygiene, and tobacco usage [10].

Candida infection in patients with malignant diseases can lead to invasive infection and candidemia. The change in the etiology of oral candidiasis from Candida albicans, the commonly encountered species, to non-Candida albicans like Candida glabrata and Candida krusei, the more inherently drug resistant species, is particularly challenging for choosing the antifungal drug. Fluconazole is the first line of drug used to treat fungal infections in head and neck cancer [11]. Increase in resistance to fluconazole is being reported among cancer patients $[1,5,12]$. In the present study we have evaluated the association of clinicomicrobiological findings among cancer patients with oral candidiasis and the antifungal susceptibility pattern of the isolates.

The common cancer type encountered was carcinoma of oral cavity followed by malignancy of gastrointestinal tract (GIT) similar to what is reported by Afraseyabi et al. [3]. Dryness of mouth and pain in the oral cavity are the most frequently encountered symptoms. In the present study we found out that there was a significant association between the presence of dry mouth and isolation of Candida species $(p<$ 0.001). Alt-Epping et al. have reported a similar association between dryness of mouth and isolation of Candida [13]. Dryness of mouth can occur as a result of chemotherapy or radiotherapy and can cause mucosal disruption facilitating infection by Candida [13]. The association between the presence of a symptom and the isolation of Candida is not causal all the time. However, from a clinical viewpoint, associating certain clinical signs and symptoms with the microbiological findings will be helpful to ascertain the affliction of the sign/symptom and a necessity to identify and treat the cause. Such associations might be useful clinically.

Studies by Nadagir et al. and Lattif et al. have showed a direct gram staining positivity rate of $75 \%$ compared to $90 \%$ in the present study $[14,15]$. Direct gram staining of the specimen along with the clinical signs and symptoms for oral candidiasis can be a valuable tool in differentiating colonisation from infection. In the present study, 95\% of the Candida albicans showed germ tube test positivity. Enwuru et al. and Srinivasan and Kenneth have reported a germ tube positivity of $96.7 \%$ and $89 \%$ among Candida albicans, respectively $[16,17]$. Although germ tube test, a simple rapid test, offers 95\% consistency for identifying Candida albicans, it must be used in concurrence with other phenotypic tests for species identification. Oral candidial infection was seen highest among patients with carcinoma of oral cavity (68) (89\%), similar to Lone et al., followed by carcinoma of gastrointestinal tract 34 (68\%). 
TABLE 5: Antifungal susceptibility pattern by Microbroth dilution method.

\begin{tabular}{|c|c|c|c|c|c|c|}
\hline $\begin{array}{l}\text { S. } \\
\text { number }\end{array}$ & Antifungal drug & $\begin{array}{l}\text { Fluconazole } \\
\text { resistant }\end{array}$ & $\begin{array}{l}\text { Fluconazole } \\
\text { SDD }^{*}\end{array}$ & $\begin{array}{l}\text { Itraconazole } \\
\text { resistant }\end{array}$ & $\begin{array}{l}\text { Itraconazole } \\
\text { SDD }\end{array}$ & $\begin{array}{c}\text { Amphotericin B } \\
\text { susceptible }\end{array}$ \\
\hline & $\mathrm{MIC}^{\S}$ range & $>8 \mu \mathrm{g} / \mathrm{mL}$ & $16-32 \mu \mathrm{g} / \mathrm{mL}$ & $>0.125 \mu \mathrm{g} / \mathrm{mL}$ & $\begin{array}{c}0.25- \\
0.5 \mu \mathrm{g} / \mathrm{mL} \\
\end{array}$ & $<1 \mu \mathrm{g} / \mathrm{mL}$ \\
\hline 1 & $\begin{array}{l}\text { Candida albicans } \\
n=94\end{array}$ & $6(5.8 \%)$ & $23(24.4 \%)$ & $12(12.7 \%)$ & $20(21.27 \%)$ & $94(100 \%)$ \\
\hline 2 & $\begin{array}{l}\text { Candida tropicalis } \\
n=34\end{array}$ & $4(11.7 \%)$ & $10(29.4 \%)$ & $4(12 \%)$ & $11(32.3 \%)$ & $34(100 \%)$ \\
\hline 3 & $\begin{array}{l}\text { Candida glabrata } \\
n=14\end{array}$ & $3(21.4 \%)$ & $4(28.5 \%)$ & $4(28.5 \%)$ & $5(35.7 \%)$ & $14(100 \%)$ \\
\hline 4 & $\begin{array}{l}\text { Candida krusei } \\
n=10\end{array}$ & $10(100 \%)$ & 0 & $4(60 \%)$ & $2(20 \%)$ & $10(100 \%)$ \\
\hline 5 & $\begin{array}{l}\text { Candida parapsilosis } \\
n=6\end{array}$ & 0 & 0 & 0 & 0 & $6(100 \%)$ \\
\hline \multirow[t]{2}{*}{6} & $\begin{array}{l}\text { Candida kefyr } \\
n=4\end{array}$ & 0 & 0 & 0 & 0 & $4(100 \%)$ \\
\hline & Overall resistance $\%$ & $23(14.1 \%)$ & $37(22.8 \%)$ & $22(14.8 \%)$ & $38(23.4 \%)$ & Nil \\
\hline
\end{tabular}

SDD* : susceptible dose dependent.

$\mathrm{MIC}^{\mathfrak{s}}$ : minimum inhibitory concentration.

Candida albicans was the predominant species isolated followed by Candida tropicalis, similar to Schelenz et al. and Safdar et al. (74\% and $67.3 \%$ were Candida albicans, resp.) $[18,19]$. Studies have reported Candida glabrata as the commonly isolated non-Candida albicans among cancer patients $[5,19]$. Oral colonisation with non-Candida albicans occurs in higher rates in cancer patients [2].

With the usage of azoles for the empirical treatment of candidial infection, there has been a rise in the incidence of non-Candida albicans like Candida krusei and Candida glabrata with reduced susceptibility to azole antifungal agents [4]. Hence, it is essential to regularly investigate the antifungal resistance pattern to get up-to-date information which will help the physician in selecting the antifungal drug for empirical therapy. The overall resistance for fluconazole and Itraconazole in the present study was $14.1 \%$ and $14.8 \%$, respectively. Literature from across the world has reported fluconazole and Itraconazole resistance to be ranging from $2 \%$ to $10 \%$ and from $9 \%$ to $10 \%$, respectively $[5,18,19]$. The resistance rate for fluconazole and Itraconazole in the present study was high compared to the above-cited studies.

In the present study, for Candida albicans the resistance for fluconazole and Itraconazole was $6 \%$ and $12.3 \%$, respectively, similar to Schelenz et al. and Safdar et al. $[18,19]$. In the present study, for Candida tropicalis, resistance for fluconazole and Itraconazole was $12 \%$. Safdar et al. has reported fluconazole and Itraconazole resistance as $19 \%$ and $21 \%$, respectively, for Candida tropicalis among cancer patients [19].

Candida glabrata showed a resistance of $21.5 \%$ and $14.3 \%$ for fluconazole and Itraconazole, respectively. In contrast to our study, Safdar et al. have reported a high resistance among Candida glabrata for fluconazole and Itraconazole $(30.8 \%$ and $46.2 \%$, resp.). Bagg et al. have reported a resistance as high as $78.7 \%$ for fluconazole among Candida glabrata [2]. For all patients with culture positivity, the antifungal susceptibility pattern was informed to the treating physician and oral fluconazole therapy was given for 112 patients along with Clotrimazole/Amphotericin B oral lozenges. In case of fluconazole resistance, Itraconazole/Clotrimazole were prescribed. Follow-up was lost for the rest of forty patients. The limitation of the present study was that the previous oral Candida colonisation status of the recruited patients prior to the initiation of chemotherapy and/or radiotherapy was not known and whether the colonised Candida species were implicated in causing the present infection was also not assessed.

In the present study, non-Candida albicans have demonstrated a higher percentage of resistance compared to Candida albicans for the empirically used drugs like fluconazole and Itraconazole. High incidence of oral colonisation and infection with such inherently drug resistant isolates becomes more challenging for choosing the prophylactic drug. Such drug resistant isolates can invade underlying mucosa and enter the blood stream causing invasive infections. Prevalence of fungal infections has increased several times among individuals with lowered immune status such as cancer patients on chemotherapy and radiotherapy [4]. Cytotoxic therapy causes dryness of oral mucosa facilitating infections by various pathogens. Studies have reported that development of candidiasis is a two-step process consisting of colonisation and subsequent invasion of epithelial layer $[4,20]$. Once colonisation has been established, impaired cellular immunity permits invasion of epithelial layer. Neutropenia, irradiation, and chemotherapy will lead to mucosal disruption facilitating deeper invasion by Candida [4].

The emergence of antifungal resistance within Candida species particularly in cancer patients is of serious concern because such drug resistant isolates may invade the deeper tissues leading to disseminated infection. The high prevalence of Candida in the oral cavity of cancer patients treated by chemotherapy/radiotherapy necessitates the need for routine 
periodic surveillance of fungal infections to determine the antifungal resistance pattern.

\section{Conclusion}

Oral candidiasis is a common fungal infection in patients with cancer on treatment with chemotherapy and/or radiotherapy. The association between clinical and microbiological findings can help in the correlation of certain symptoms with the isolation of Candida among cancer patients in certain instances, but such associations are not always causal. From a clinical perspective it might be useful if the patient complains of that particular symptom like dry mouth; alertness for associated Candida infection should be high. Candida albicans and non-Candida albicans differ significantly in their antifungal susceptibility pattern. Non-Candida albicans like Candida krusei are inherently resistant to azoles. Hence, species level identification with the in vitro antifungal susceptibility pattern is essential to choose the appropriate antifungal drug and to predict the outcome of therapy.

\section{Disclosure}

This study is associated with Department of Microbiology, Government Kilpauk Medical College, Chennai, Tamil Nadu.

\section{Competing Interests}

The authors declare that they have no competing interests.

\section{References}

[1] M. S. Lone, G. Bashir, N. Bali et al., "Oral Candida colonization and infection in cancer patients and their antifungal susceptibility in a tertiary care hospital," International Journal of Advanced Research, vol. 2, no. 5, pp. 541-550, 2014.

[2] J. Bagg, M. P. Sweeney, M. A. O. Lewis et al., "High prevalence of non-albicans yeasts and detection of anti-fungal resistance in the oral flora of patients with advanced cancer," Palliative Medicine, vol. 17, no. 6, pp. 477-481, 2003.

[3] Sh. Afraseyabi, A. Afkhamzadeh, H. Sabori, F. Verdi, N. Khaksar, and B. Mosavei, "Oral candidiasis amongst cancer patients at Qods hospitals in Sanandaj," African Journal of Clinical and Experimental Microbiology, vol. 12, no. 3, pp. 1595-1689, 2011.

[4] R. V. Lalla, M. C. Latortue, C. H. Hong et al., "A systematic review of oral fungal infections in patients receiving cancer therapy," Supportive Care in Cancer, vol. 18, no. 8, pp. 985-992, 2010.

[5] T. Shokohi, Z. Bandalizadeh, M. T. Hedayati, and S. Mayahi, "In vitro antifungal susceptibility of Candida species isolated from oropharyngeal lesions of patients with cancer to some antifungal agents," Jundishapur Journal of Microbiology, vol. 4, no. 1, pp. S19-S26, 2011.

[6] R. Adhikary and S. Joshi, "Species distribution and anti-fungal susceptibility of Candidaemia at a multi super-specialty center in Southern India," Indian Journal of Medical Microbiology, vol. 29, no. 3, pp. 309-311, 2011.

[7] D. J. Diekema, S. A. Messer, L. B. Boyken et al., "In vitro activity of seven systemically active antifungal agents against a large global collection of rare Candida species as determined by CLSI broth microdilution methods," Journal of Clinical Microbiology, vol. 47, no. 10, pp. 3170-3177, 2009.

[8] Clinical and Laboratory Standards Institute (CLSI), "Reference method for broth dilution antifungal susceptibility testing of yeasts. Approved standard, 3rd ed.," CLSI Document M27-A3, Clinical and Laboratory Standards Institute, Wayne, Pa, USA, 2009.

[9] V. Ramirez-Amador, S. Silverman Jr., P. Mayer, M. Tyler, and J. Quivey, "Candidal colonization and oral candidiasis in patients undergoing oral and pharyngeal radiation therapy," Oral Surgery, Oral Medicine, Oral Pathology, Oral Radiology, and Endodontics, vol. 84, no. 2, pp. 149-153, 1997.

[10] R.-J. Bensadoun, L. L. Patton, R. V. Lalla, and J. B. Epstein, "Oropharyngeal candidiasis in head and neck cancer patients treated with radiation: update 2011," Supportive Care in Cancer, vol. 19, no. 6, pp. 737-744, 2011.

[11] S. W. Redding, R. C. Zellars, W. R. Kirkpatrick et al., "Epidemiology of oropharyngeal Candida colonization and infection in patients receiving radiation for head and neck cancer," Journal of Clinical Microbiology, vol. 37, no. 12, pp. 3896-3900, 1999.

[12] A. Mañas, L. Cerezo, A. De La Torre et al., "Epidemiology and prevalence of oropharyngeal candidiasis in Spanish patients with head and neck tumors undergoing radiotherapy treatment alone or in combination with chemotherapy," Clinical and Translational Oncology, vol. 14, no. 10, pp. 740-746, 2012.

[13] B. Alt-Epping, R. K. Nejad, K. Jung, U. Groß, and F. Nauck, "Symptoms of the oral cavity and their association with local microbiological and clinical findings-a prospective survey in palliative care," Supportive Care in Cancer, vol. 20, no. 3, pp. 531537, 2012.

[14] S. D. Nadagir, S. K. Chunchanur, L. H. Halesh, K. Yasmeen, M. R. Chandrasekhar, and B. S. Patil, "Significance of isolation and drug susceptibility testing of non-Candida albicans species causing oropharyngeal Candidiasis in HIV patients," Southeast Asian Journal of Tropical Medicine and Public Health, vol. 39, no. 3, pp. 492-495, 2008.

[15] A. A. Lattif, U. Banerjee, R. Prasad et al., "Susceptibility pattern and molecular type of species-specific candida in oropharyngeal lesions of indian human immunodeficiency virus-positive patients," Journal of Clinical Microbiology, vol. 42, no. 3, pp. 1260-1262, 2004.

[16] C. A. Enwuru, A. Ogunledun, N. Idika et al., "Fluconazole resistant opportunistic oro-pharyngeal candida and non-candida yeast-like isolates from HIV infected patients attending ARV clinics in Lagos, Nigeria," African Health Sciences, vol. 8, no. 3, pp. 142-148, 2008.

[17] L. Srinivasan and J. Kenneth, "Antibiotic susceptibility of Candida isolates in a tertiary care hospital in Southern India," Indian Journal of Medical Microbiology, vol. 24, no. 1, pp. 80-81, 2006.

[18] S. Schelenz, S. Abdallah, G. Gray et al., "Epidemiology of oral yeast colonization and infection in patients with hematological malignancies, head neck and solid tumors," Journal of Oral Pathology and Medicine, vol. 40, no. 1, pp. 83-89, 2011.

[19] A. Safdar, V. Chaturvedi, E. W. Cross et al., "Prospective study of Candida species in patients at a comprehensive cancer center," Antimicrobial Agents and Chemotherapy, vol. 45, no. 7, pp. 21292133, 2001.

[20] C. E. Delsing, C. P. Bleeker-Rovers, F. L. van de Veerdonk et al., "Association of esophageal candidiasis and squamous cell carcinoma," Medical Mycology Case Reports, vol. 1, no. 1, pp. 5-8, 2012. 


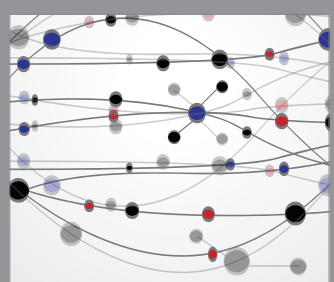

The Scientific World Journal
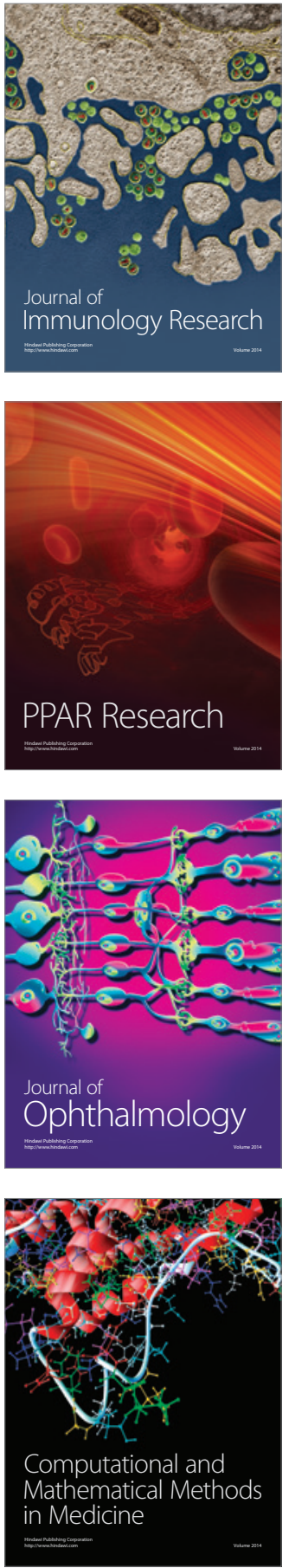

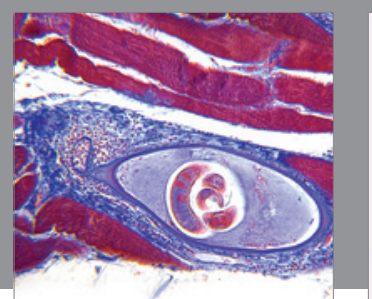

Gastroenterology Research and Practice

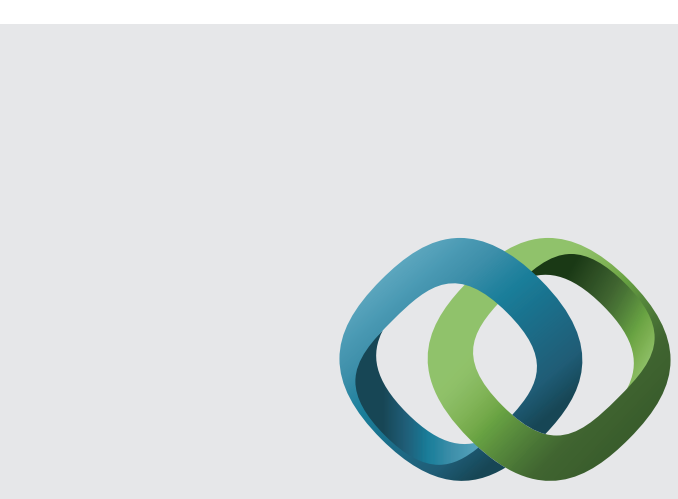

\section{Hindawi}

Submit your manuscripts at

http://www.hindawi.com
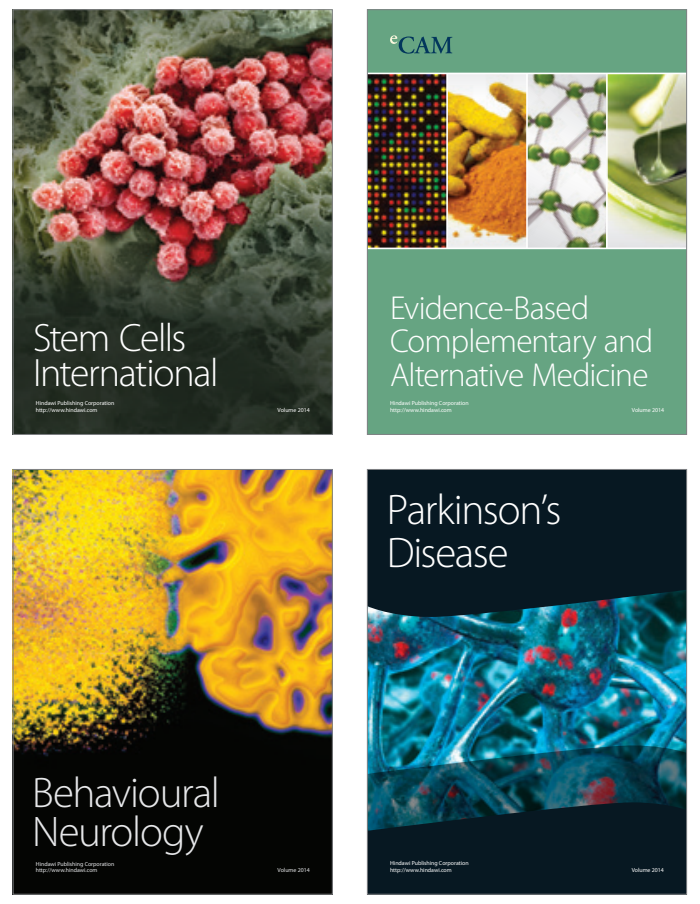
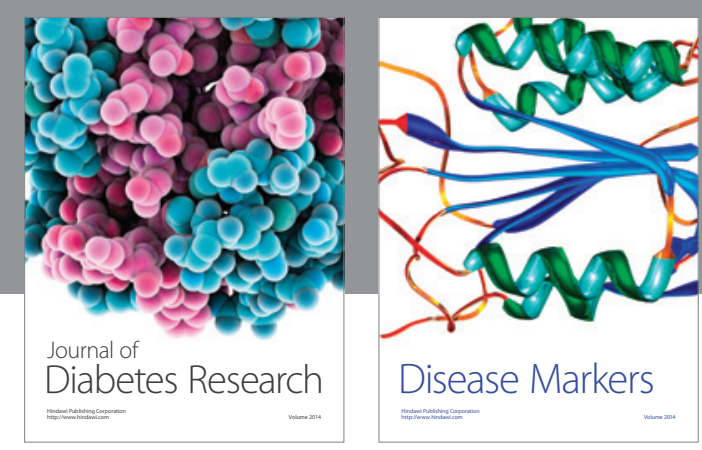

Disease Markers
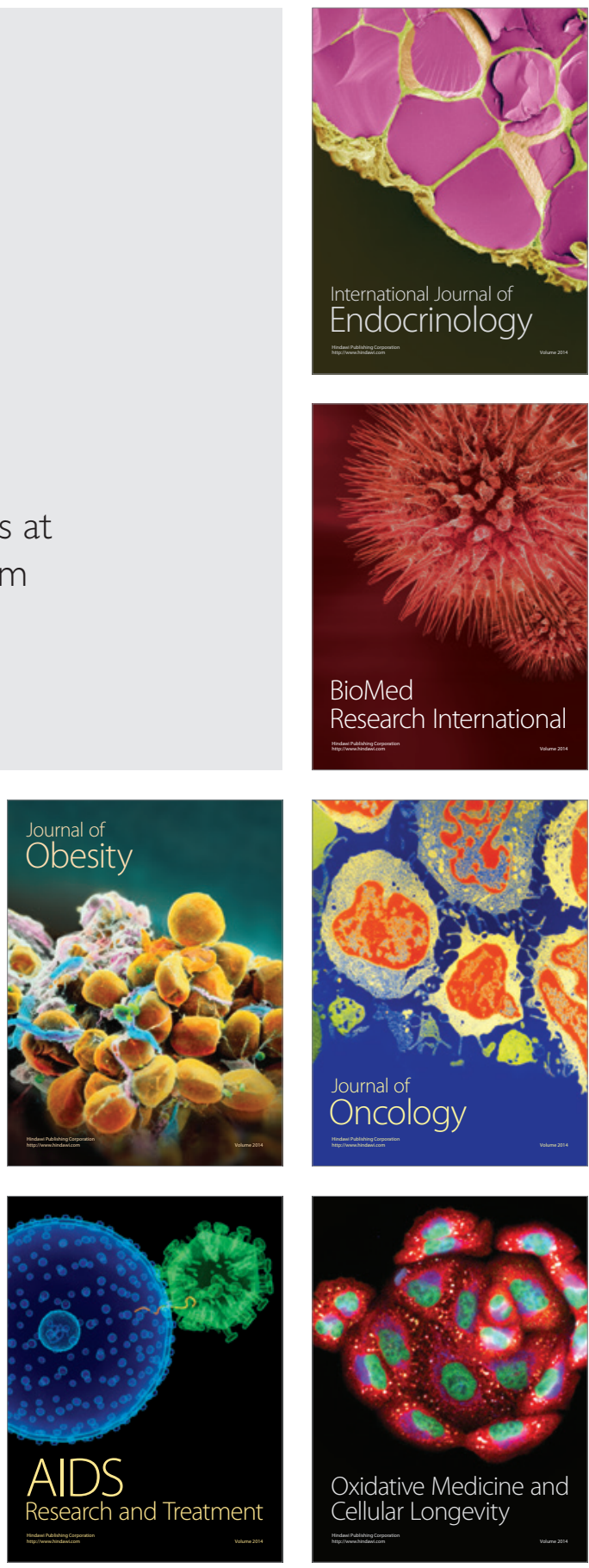